\title{
1 16S rRNA Gene Amplicon Profiling of the New Zealand parasitic blowfly
}

\section{Calliphora vicina.}

3 Nikola Palevich, Paul H. Maclean, Luis Carvalho and Ruy Jauregui.

4 AgResearch Limited, Grasslands Research Centre, Palmerston North, New Zealand.

$5 \quad$ Corresponding author: E-mail: nik.palevich@agresearch.co.nz.

\section{ABSTRACT}

7 Here, we present a 16S rRNA gene amplicon sequence data set and profiles demonstrating the bacterial diversity

8 of larval and adult Calliphora vicina, collected from Ashhurst, New Zealand (May 2020). The three dominant

9 genera among adult male and female C. vicina were Serratia and Morganella (phylum Proteobacteria) and

10 Carnobacterium (phylum Firmicutes), while the larvae were also dominated by the genera Lactobacillus (phylum

11 Firmicutes).

\section{ANNOUNCEMENT}

13 Ectoparasitic flies (blowflies) are a significant animal welfare and production issue for farmers worldwide (1).

14 Control of blowflies is problematic because the flies are unpredictable and highly mobile, and strike (or myiasis)

15 is difficult to see initially but has an immediate impact on animal production and welfare. Currently control relies

16 heavily on the prophylactic application of long-acting chemicals to all sheep, but this approach is increasingly

17 under threat due to development of resistance to current treatments $(2,3)$. Calliphora vicina NZ_CalVic_NP (4,

18 5) was selected for microbiome assessment as a representative of a New Zealand field strain of $C$. vicina. In this

19 study, we have investigated the larval, adult male and adult female bacterial microbial profiles of $C$. vicina to gain

20 a better understanding of the microbial communities of blowflies targeting the development of new interventions

21 such as probiotics, bioactive compounds, vaccines or insecticides. 
The C. vicina specimen larvae were collected from a farm site in Ashhurst area in New Zealand $\left(40^{\circ} 18^{\prime} \mathrm{S}, 175^{\circ} 45^{\prime}\right.$

E). Lab reared blowflies were maintained on beef liver as protein source and a $10 \%$ sugar solution, with the procedures for blowfly propagation and sample preparation were based done according to Dear J.P. (1985). To

remove surface adherent bacteria from lab reared C. vicina, pools of larvae, entire adult male and adult female

were separated and washed twice in sterile phosphate-buffered saline (PBS, $\mathrm{pH}$ 7.4), snap frozen in liquid nitrogen, and transferred to $-80{ }^{\circ} \mathrm{C}$ storage prior to DNA extraction. Genomic DNA for metagenomic $16 \mathrm{~S}$ rRNA gene amplicon sequencing of the V3-V4 hypervariable region was isolated from C. vicina pooled samples of 100 larvae as well as 10 entire adult males and females per replicate ( $n=5$ for each). High molecular weight genomic DNA was prepared using a modified phenol:chloroform protocol recently applied to difficult samples such as parasitic roundworms $(7,8)$, fastidious anaerobic rumen bacteria (9-11) and spore-forming psychrotolerant Clostridium isolated from spoiled meat $(12,13)$. A DNA library was prepared using the Illumina $16 \mathrm{~S}$ V3-V4 rRNA library preparation method (Illumina, Inc., San Diego, CA) according to the manufacturer's instructions (20), and sequenced on the Illumina MiSeq platform with the $2 \times 250$ bp paired-end (PE) reagent kit v2 producing a total of 3,017,007 PE raw reads.

The processing of the amplicon reads followed a modified form of the pipeline described in (21). The reads produced by the sequencing instrument were paired using the program FLASH2 (22). Paired reads were then quality trimmed using Trimmomatic 0.38 (23). The trimmed reads were reformatted as fasta, and the read headers were modified to include the sample name. All reads were compiled in a single file, and the Mothur (24) program suite was used to remove reads with homopolymers longer than $10 \mathrm{nt}$ and to collapse the reads into unique representatives. The collapsed reads were clustered using the program Swarm (25). The clustered reads were filtered based on their abundance, keeping representatives that were a) present in one sample with a relative abundance $>0.1 \%, \mathrm{~b}$ ) present in $>2 \%$ of the samples with a relative abundance $>0.01 \%$ or c) present in $5 \%$ of the samples at any abundance level. The selected representatives were annotated using Qiime (26) with the Silva database v138 (27). The annotated tables were then used for downstream statistical analysis. The predominant 
46 phyla in all samples were Proteobacteria (Fig. 1) and at the genus level, Serratia, Morganella and

47 Carnobacterium, while the larvae were also dominated by Lactobacillus (phylum Firmicutes).

48 The metagenomic 16S rRNA gene amplicon sequencing of $C$. vicina field strain NZ_CalVic_NP reported here is

49 a valuable resource for future studies investigating the bacterial genetic mechanisms associated with flystrike.

50 Management of flystrike in a world increasingly demanding fewer inputs of synthetic chemicals to food producing

51 animals will be challenging. Equally, this research is important owing to the diminished efficacy demonstrated

52 by current blowfly treatments due to the emergence of resistance in blowflies against many classes of insecticides.

53 Data availability. The $16 \mathrm{~S}$ rRNA gene amplicon sequence data have been deposited in the GenBank Sequence

54 Read Archive (SRA) under the BioProject accession number PRJNA667961.

\section{ACKNOWLEDGEMENTS}

56 We thank Xiaoxiao Lin for assistance with the DNA sequencing and Paul Candy for sample collection. This

57 research was supported by the Agricultural and Marketing Research and Development Trust (AGMARDT)

58 Postdoctoral Fellowships Programme [No. P17001] and the AgResearch Ltd Strategic Science Investment Fund

59 (SSIF) [No. PRJ0098715] of New Zealand. 
bioRxiv preprint doi: https://doi.org/10.1101/2021.03.12.435192; this version posted March 13, 2021. The copyright holder for this preprint (which was not certified by peer review) is the author/funder, who has granted bioRxiv a license to display the preprint in perpetuity. It is made available under aCC-BY-NC-ND 4.0 International license.

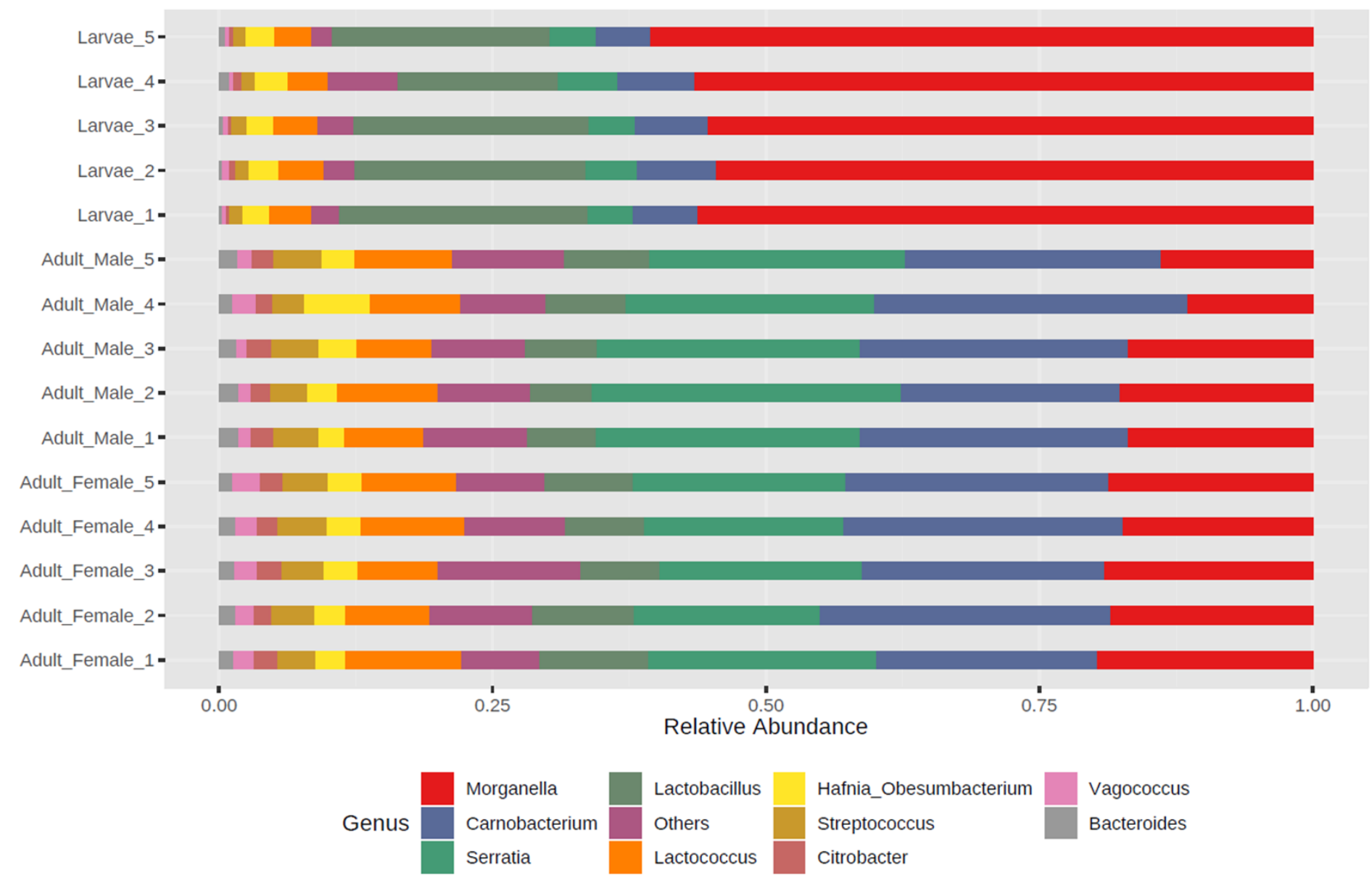

61 FIG 1. The taxonomic composition of the dominant bacteria of New Zealand C. vicina. Relative abundance of 62 the dominant bacterial genera obtained from $16 \mathrm{~S}$ rRNA sequencing of $C$. vicina field strain NZ_CalVic_NP 63 larvae, adult males and female samples. Genera with a relative abundance of less than $1 \%$ and unassigned 64 amplicon sequence variants were grouped together as Others. 


\section{REFERENCES}

1. Githeko AK, Lindsay SW, Confalonieri UE, Patz JA. 2000. Climate change and vector-borne diseases: a regional analysis. Bulletin of the World Health Organization 78:1136-1147.

2. Hall M, Wall R. 1995. Myiasis of humans and domestic animals. Advances in parasitology 35:257-334.

3. Fischer O, Matlova L, Dvorska L, Svastova P, Bartl J, Weston R, Pavlik I. 2004. Blowflies Calliphora vicina and Lucilia sericata as passive vectors of Mycobacterium avium subsp. avium, M. a. paratuberculosis and M. a. hominissuis. Medical and veterinary entomology 18:116-122.

4. Palevich N, Carvalho L, Maclean P. 2020. Characterization of the complete mitochondrial genome of the New Zealand parasitic blowfly <em>Calliphora vicina</em> (Insecta: Diptera: Calliphoridae). bioRxiv doi:10.1101/2020.11.05.370361:2020.2011.2005.370361.

5. Palevich N, Carvalho, Luis, Maclean, Paul. 2020. The Complete Mitochondrial Genome of the New Zealand Parasitic Blowfly Lucilia sericata (Insecta: Diptera: Calliphoridae). Preprints 2020100601.

6. Dear JP. 1986. Calliphoridae (Insecta: Diptera). Fauna of New Zealand, 8: 88.

7. Palevich N, Maclean PH, Baten A, Scott RW, Leathwick DM. 2019. The Genome Sequence of the Anthelmintic-Susceptible New Zealand Haemonchus contortus. Genome biology and evolution 11:19651970.

8. Palevich N, Maclean PH, Choi Y-J, Mitreva M. 2020. Characterization of the Complete Mitochondrial Genomes of Two Sibling Species of Parasitic Roundworms, Haemonchus contortus and Teladorsagia circumcincta. Frontiers in Genetics 11.

9. Palevich N, Kelly WJ, Ganesh S, Rakonjac J, Attwood GT. 2019. Butyrivibrio hungatei MB2003 Competes Effectively for Soluble Sugars Released by Butyrivibrio proteoclasticus B316 ${ }^{\mathrm{T}}$ during Growth on Xylan or Pectin. Applied and Environmental Microbiology 85:e02056-02018.

10. Palevich N, Kelly WJ, Leahy SC, Denman S, Altermann E, Rakonjac J, Attwood GT. 2019. Comparative genomics of rumen Butyrivibrio spp. uncovers a continuum of polysaccharide-degrading capabilities. Applied and environmental microbiology 86.

11. Palevich N, Maclean PH, Kelly WJ, Leahy SC, Rakonjac J, Attwood GT. 2020. Complete Genome Sequence of the Polysaccharide-Degrading Rumen Bacterium Pseudobutyrivibrio xylanivorans MA3014 Reveals an Incomplete Glycolytic Pathway. Genome Biology and Evolution 12:1566-1572.

12. Palevich N, Palevich FP, Maclean PH, Altermann E, Gardner A, Burgess S, Mills J, Brightwell G. 2021. Comparative genomics of Clostridium species associated with vacuum-packed meat spoilage. Food Microbiology 95:103687.

13. Palevich N, Palevich FP, Maclean PH, Jauregui R, Altermann E, Mills J, Brightwell G. 2019. Draft genome sequence of Clostridium estertheticum subsp. laramiense DSM $14864^{\mathrm{T}}$, isolated from spoiled uncooked beef. Microbiology resource announcements 8.

14. Kozich JJ, Westcott SL, Baxter NT, Highlander SK, Schloss PD. 2013. Development of a dual-index sequencing strategy and curation pipeline for analyzing amplicon sequence data on the MiSeq Illumina sequencing platform. Applied and environmental microbiology 79:5112-5120.

15. Camarinha-Silva A, Jáuregui R, Pieper DH, Wos-Oxley ML. 2012. The temporal dynamics of bacterial communities across human anterior nares. Environmental microbiology reports 4:126-132.

16. Magoč T, Salzberg SL. 2011. FLASH: fast length adjustment of short reads to improve genome assemblies. Bioinformatics 27:2957-2963.

17. Bolger AM, Lohse M, Usadel B. 2014. Trimmomatic: a flexible trimmer for Illumina sequence data. Bioinformatics 30:2114-2120.

18. Schloss PD, Westcott SL, Ryabin T, Hall JR, Hartmann M, Hollister EB, Lesniewski RA, Oakley BB, Parks DH, Robinson CJ. 2009. Introducing mothur: open-source, platform-independent, communitysupported software for describing and comparing microbial communities. Applied and environmental microbiology 75:7537-7541. 
19. Mahé F, Rognes T, Quince C, de Vargas C, Dunthorn M. 2014. Swarm: robust and fast clustering method for amplicon-based studies. PeerJ 2:e593.

20. Caporaso JG, Kuczynski J, Stombaugh J, Bittinger K, Bushman FD, Costello EK, Fierer N, Pena AG, Goodrich JK, Gordon JI. 2010. QIIME allows analysis of high-throughput community sequencing data. Nature methods 7:335-336.

21. Quast C, Pruesse E, Yilmaz P, Gerken J, Schweer T, Yarza P, Peplies J, Glöckner FO. 2012. The SILVA ribosomal RNA gene database project: improved data processing and web-based tools. Nucleic acids research 41:D590-D596. 